PROCEEDINGS OF THE

AMERICAN MATHEMATICAL SOCIETY

Volume 128, Number 4, Pages 963-973

S 0002-9939(99)05080-7

Article electronically published on July 28, 1999

\title{
HILBERT COEFFICIENTS AND THE ASSOCIATED GRADED RINGS
}

\author{
HSIN-JU WANG
}

(Communicated by Wolmer V. Vasconcelos)

\begin{abstract}
Let $(R, \mathfrak{m})$ be a $d$-dimensional Cohen-Macaulay local ring with infinite residue field. Let $I$ be an $\mathfrak{m}$-primary ideal of $R$. In this paper, we prove that if $\sum_{n=1}^{\infty} \lambda\left(I^{n} / I^{n-1} J\right)-e_{1}(I)=1$ for some minimal reduction $J$ of $I$, then $\operatorname{depth} G(I) \geq d-2$.
\end{abstract}

\section{INTRODUCTION}

Let $(R, \mathfrak{m})$ be a $d$-dimensional Cohen-Macaulay local ring with infinite residue field and $I$ be an $\mathfrak{m}$-primary ideal of $R$. Let $G(I)=\bigoplus_{n>0} I^{n} / I^{n+1}$ be the associated graded ring of $R$. During the past years, many commutative algebraists tried to estimate the depth of $G(I)$ for ideals $I$ having good properties. In 1978, Valabrega and Valla obtained in 6] that $G(I)$ is Cohen-Macaulay if and only if there exists a minimal reduction $J$ of $I$ such that $I^{n} \cap J=I^{n-1} J$ for all $n$. Later on, Guerrieri studied the so called Valabrega-Valla module and made the following conjecture in her paper [1].

Conjecture 1. If $\sum_{n=1}^{\infty} \lambda\left(I^{n} \cap J / I^{n-1} J\right)=t$ for some minimal reduction $J$ of $I$, then depth $G(I) \geq d-t$.

On the other hand, Sally in [5] studied the depth of $G(\mathfrak{m})$ by considering the classical bound of Abhyankar on the multiplicity $e$ of $R$; namely, $e \geq \mu(\mathfrak{m})-d+1$, where $\mu(I)$ stands for the minimal number of a generating set of $I$. She first studied the case of rings with minimal multiplicity, i.e., $e=\mu(\mathfrak{m})-d+1$, then the cases $e-(\mu(\mathfrak{m})-d+1)=1,2$. Recently, Huckaba and Marley showed in 4] that if one considers the first coefficient $e_{1}(I)$, then $e_{1}(I)$ is bounded above by $\sum_{n=1}^{\infty} \lambda\left(I^{n} / I^{n-1} J\right)$ for any minimal reduction $J$ of $I$, and later Huckaba and Vaz Pinto independently showed that depth $G(I) \geq d-1$ if $e_{1}(I)=\sum_{n=1}^{\infty} \lambda\left(I^{n} / I^{n-1} J\right)$ for some minimal reduction $J$ of $I$.

Received by the editors October 3, 1997 and, in revised form, May 19, 1998.

1991 Mathematics Subject Classification. Primary 13A30, 13D40, 13H10.

Key words and phrases. Hilbert coefficient, associated graded ring. 
In a similar fashion to what Sally did with the Abhyankar's bound, we can raise the following conjecture on the depth of $G(I)$ by considering the difference of $e_{1}(I)$ and $\sum_{n=1}^{\infty} \lambda\left(I^{n} / I^{n-1} J\right)$.

Conjecture 2. If $\sum_{n=1}^{\infty} \lambda\left(I^{n} / I^{n-1} J\right)-e_{1}(I)=t$, then depth $G(I) \geq d-1-t$.

One can see in section 2 that Conjecture 1 holds if we can give an affirmative answer to Conjecture 2. In this paper, we are able to show, by using a method developed in [8] concerning the Sally module defined in [7], that if $\sum_{n=1}^{\infty} \lambda\left(I^{n} / I^{n-1} J\right)-$ $e_{1}(I)=1$, then depth $G(I) \geq d-2$. Hence the Conjecture 1 holds if $t \leq 2$.

\section{Preliminaries}

Throughout, let $(R, \mathfrak{m})$ be a Cohen-Macaulay local ring with infinite residue field. Let $I$ be an $\mathfrak{m}$-primary ideal of $R$ and $J$ a minimal reduction of $I$. Let $G(I)$ be the associated graded ring of $R$. An element $x \in I \backslash I^{2}$ is called superficial for $I$ if $\left(0: G(I) x^{*}\right)_{n}=0$ for all $n$ sufficiently large. Here, $x^{*}$ denotes the image of $x$ in $I / I^{2} \subseteq G(I)$. A sequence $x_{1}, \ldots, x_{k}$ is called superficial sequence for $I$ if $x_{1}$ is superficial for $I$ and $x_{i}$ is superficial for $I /\left(x_{1}, \ldots, x_{i-1}\right)$. In [3], Huckaba proved that if $\operatorname{dim} R=1$, then $e_{1}(I)=\sum_{n=1}^{\infty} \lambda\left(I^{n} / I^{n-1} J\right)$ for any minimal reduction $J$ of $I$; therefore it is easy to see the following:

Lemma 2.1. If $\operatorname{dim} R=d$ and $x_{1}, \ldots, x_{d-1} \in J$ is a superficial sequence for $I$, then

$$
e_{1}(I)=\sum_{n=1}^{\infty} \lambda\left(I^{n} /\left(I^{n-1} J+I^{n} \cap\left(x_{1}, \ldots, x_{d-1}\right)\right)\right) .
$$

In 4], Huckaba and Marley gave in Lemma 2.2 a sufficient conditions for $G(I)$ having positive depth. We restate it here in the following special form.

Lemma 2.2. Let $x \in J$ be a superficial element for I. If depth $G(I /(x))>0$, then depth $G(I)>0$.

Corollary 2.3. Let $(R, \mathfrak{m})$ be a 3-dimensional Cohen-Macaulay local ring with infinite residue field. Let $I$ be an $\mathfrak{m}$-primary ideal of $R$ and $J$ be a minimal reduction of I. Suppose that

$$
\sum_{n=1}^{\infty} \lambda\left(I^{n} / I^{n-1} J\right)-e_{1}(I)=1 .
$$

Let $x \in J$ be a superficial element for I. If $\sum_{n=1}^{\infty} \lambda\left(I^{n} /\left(I^{n-1} J+I^{n} \cap(x)\right)\right)=e_{1}(I)$, then depth $G(I)>0$.

Proof. The conclusion follows from Lemma 2.2 and the fact ([3, Theorem 3.1]) that if $\sum_{n=1}^{\infty} \lambda\left(I^{n} / I^{n-1} J\right)=e_{1}(I)$, then depth $G(I) \geq \operatorname{dim} R-1$. 
The following two lemmas are easy to derive; we leave the proofs to the reader.

Lemma 2.4. Let $(R, \mathfrak{m})$ be a 3-dimensional Cohen-Macaulay local ring with infinite residue field. Let $I$ be an $\mathfrak{m}$-primary ideal of $R$ and $J=\left(x_{1}, x_{2}, x_{3}\right)$ be a minimal reduction of $I$. Let $N \geq 2$. Suppose that $I^{n} \cap\left(x_{i}, x_{j}\right) \subseteq I^{n-1} J \forall n<N$ and $\forall i, j \in\{1,2,3\}$. Then $\forall n<N$ and $\forall m \geq 1$,

(1) $I^{n}: x_{i}=I^{n-1} \forall i$.

(2) $I^{n} J^{m}: x_{i}=I^{n} J^{m-1} \forall i$.

(3) $I^{n}: x_{2}=I^{n}: x_{3}=I^{n-1}\left(\bmod x_{1}\right)$.

(4) $I^{n} J^{m}: x_{2}=I^{n} J^{m}: x_{3}=I^{n} J^{m-1}\left(\bmod x_{1}\right)$.

(5) Let $\lambda_{0}, \ldots, \lambda_{t}$ be either unit or 0 but not all 0 . Let $s \in R$ be such that $s\left(\sum_{i=0}^{t} \lambda_{i} x_{1}^{n-i} x_{2}^{i}\right) \in I^{n} J^{m}$. Then $s \in I^{n} J^{m-t}$ if $m \geq t$ or $s \in I^{n-t+m}$ if $m<t$.

If, moreover, $I^{N} \cap\left(x_{1}\right) \subseteq I^{N-1} J$, then $I^{N}: x_{1}=I^{N-1}$.

Lemma 2.5. Let $N \geq 2$. If $a_{1}, \ldots, a_{n} \in I^{N}$ not all in $I^{N-1} J$, then there are only finite number of units $\lambda$ such that $\sum_{i=1}^{n} a_{i} \lambda^{i-1} \in I^{N-1} J$.

The following proposition presents a relation between the two conjectures stated in the previous section.

Proposition 2.6. If Conjecture 2 has a positive answer, then so does Conjecture 1.

Suppose that Conjecture 2 holds. Let $I$ be an $\mathfrak{m}$-primary ideal of $R$ and let $J$ be a minimal reduction of $I$. Let $t=\sum_{n=1}^{\infty} \lambda\left(\frac{I^{n} \cap J}{I^{n-1} J}\right)$. Then, by Lemma 2.1] for any superficial sequence $x_{1}, \ldots, x_{d-1} \in J$ for $I$,

$$
\begin{aligned}
\sum_{n=1}^{\infty} \lambda\left(I^{n} / I^{n-1} J\right)-e_{1}(I) & =\sum_{n=1}^{\infty} \lambda\left(\frac{I^{n} \cap\left(x_{1}, \ldots, x_{d-1}\right)+I^{n-1} J}{I^{n-1} J}\right) \\
& \leq \sum_{n=1}^{\infty} \lambda\left(\frac{I^{n} \cap J}{I^{n-1} J}\right)=t .
\end{aligned}
$$

Let $k$ be the least integer such that $\lambda\left(\frac{I^{k} \cap J}{I^{k-1} J}\right) \neq 0$. Then, by [2, Lemma 3.1], $\lambda\left(\frac{I^{k} \cap\left(x_{1}, \ldots, x_{d-1}\right)+I^{k-1} J}{I^{k-1} J}\right)<\lambda\left(\frac{I^{k} \cap J}{I^{k-1} J}\right)$, so that $\sum_{n=1}^{\infty} \lambda\left(I^{n} / I^{n-1} J\right)-e_{1}(I) \leq$ $t-1$. Therefore, depth $G(I) \geq d-t$ by assumption. This shows that Conjecture 1 holds.

\section{MAIN THEORY}

The goal of this section is to prove the following:

Theorem 3.1. Let $(R, \mathfrak{m})$ be a Cohen-Macaulay local ring of dimension $d \geq 2$ with infinite residue field. Let $I$ be an $\mathfrak{m}$-primary ideal of $R$. Suppose that there is a minimal reduction $J$ of $I$ such that $\sum_{n=0}^{\infty} \lambda\left(I^{n+1} / I^{n} J\right)-e_{1}(I)=1$. Then depth $G(I) \geq d-2$. 
By [5], it suffices to consider the case $d=3$, so we assume in the following that $d=3$. We also assume now that Theorem 3.1 doesn't hold. We shall reach a contradiction later.

Let $x_{1}, x_{2} \in J$ be a superficial sequence of $I$; then, by Corollary [2.3, we have for $i=1,2$,

$$
\sum_{n=1}^{\infty} \lambda\left(I^{n} /\left(I^{n-1} J+I^{n} \cap\left(x_{i}\right)\right)\right)-e_{1}(I)=1 .
$$

Moreover, by Lemma 2.1, we have

$$
\sum_{n=1}^{\infty} \lambda\left(I^{n} /\left(I^{n-1} J+I^{n} \cap\left(x_{1}, x_{2}\right)\right)\right)-e_{1}(I)=0 .
$$

Let $\{x, y, z\}$ be a minimal generating set of $J$. Consider the exact sequence:

$$
0 \longrightarrow T_{k, n} \longrightarrow \bigoplus^{\left(\begin{array}{c}
n+2 \\
2
\end{array}\right)} I^{k} / I^{k-1} J \stackrel{\phi_{n}}{\longrightarrow} S_{k, n}=I^{k} J^{n} / I^{k-1} J^{n+1} \longrightarrow 0
$$

where $\phi_{n}=\left(x^{n}, x^{n-1} y, x^{n-1} z, \ldots, z^{n}\right)$ and $T_{k, n}=k e r\left(\phi_{n}\right)$. From the proof of [8] Theorem 2.4], we see that there is an unique integer $N \geq 2$ such that $T_{N, n} \neq 0$ for some positive integer $n$. Notice that $N$ is independent of the choice of a generating set of $J$ since $S_{k, n}$ and $I^{k} / I^{k-1} J$ are. As $R / \mathfrak{m}$ is infinite, we may, after elementary transformation of $x, y$ and $z$, require that $\{x, y, z\}$ satisfies the following conditions.

Proposition 3.2. There is a generating set $\{x, y, z\}$ of J satisfying the following conditions:

(i) $\{x, y\},\{x, z\},\{y, z\}$ and $\{z\}$ are all superficial sequences for $I$.

(ii) $I^{n} \cap(x), I^{n} \cap(y)$ and $I^{n} \cap(z)$ are all contained in $I^{n-1} J \forall n$.

(iii) $I^{n} \cap(x, y), I^{n} \cap(x, z)$ and $I^{n} \cap(y, z)$ are all contained in $I^{n-1} J \forall n \neq N$. Moreover,

$$
\begin{aligned}
\lambda\left(\frac{I^{N} \cap(x, y)+I^{N-1} J}{I^{N-1} J}\right) & =\lambda\left(\frac{I^{N} \cap(x, z)+I^{N-1} J}{I^{N-1} J}\right) \\
& =\lambda\left(\frac{I^{N} \cap(y, z)+I^{N-1} J}{I^{N-1} J}\right)=1 .
\end{aligned}
$$

Proof. (Sketch.) Notice that (ii) follows from (i) and (11); therefore we need only to show (i) and (iii).

Let $\{x, y, z\}$ be a generating set of $J$. Let $n$ be an integer such that $T_{N, n} \neq$ 0. Then there are $a_{i j k} \in I^{N}$ not all in $I^{N-1} J$ such that $\sum_{i+j+k=n} a_{i j k} x^{i} y^{j} z^{k} \in$ $I^{N-1} J^{n+1}$. By Lemma [2.5, we may, after elementary transformation of $x, y$ and $z$, assume that $a_{n 00}, a_{0 n 0}$ and $a_{00 n}$ are not in $I^{N-1} J$.

Next, we can use prime avoidance and Corollary [2.3] to replace $\{x, y, z\}$ by elements of the set $\{x+\alpha y+\beta z\}$ so that the condition (i) holds without changing the condition that the coefficients of $x^{n}, y^{n}$ and $z^{n}$ are not in $I^{N-1} J$.

Since $\sum_{i+j+k=n} a_{i j k} x^{i} y^{j} z^{k} \in\left(I^{N-1} J\right) J^{n}$, there are $a_{1}, a_{2}, a_{3} \in I^{N-1} J$ such that $a_{n 00}-a_{1} \in I^{N} \cap(y, z), a_{0 n 0}-a_{2} \in I^{N} \cap(x, z)$ and $a_{00 n}-a_{3} \in I^{N} \cap(x, y)$. Thus the condition (iii) holds by condition (i) and (2) . 
Let $\{x, y, z\}$ be a generating set of $J$ satisfying the conditions of Proposition 3.2 Let $t$ be chosen least such that $T_{N, n} \neq 0$ for $n \geq t$. Then there are elements $\left\{a_{i j k} \mid i+j+k=t\right\}$ not all in $I^{N-1} J$ such that $\sum a_{i j k} x^{i} y^{j} z^{k} \in I^{N-1} J^{t+1}$. If $\left\{a_{0 j k} \mid j+k=t\right\} \subseteq I^{N-1} J$, then $x\left(\sum a_{i j k} x^{i-1} y^{j} z^{k}\right) \in I^{N-1} J^{t+1}$, so that by Lemma 2.4. $\sum a_{i j k} x^{i-1} y^{j} z^{k} \in I^{N-1} J^{t}$; it follows that $T_{N, t-1} \neq 0$, which contradicts the choice of $t$. Therefore, $\left\{a_{0 j k} \mid j+k=t\right\}$ are not all in $I^{N-1} J$.

Let the overbars denote $\bmod (x)$ in the following. Consider the exact sequence

$$
0 \longrightarrow \bar{T}_{k, n} \longrightarrow \bigoplus^{n+1} \bar{I}^{k} / \bar{I}^{k-1} \bar{J} \stackrel{\bar{\phi}_{n}}{\longrightarrow} \bar{I}^{k} \bar{J}^{n} / \bar{I}^{k-1} \bar{J}^{n+1} \longrightarrow 0
$$

where $\bar{\phi}_{n}=\left(\bar{y}^{n}, \bar{y}^{n-1} \bar{z}, \ldots, \bar{z}^{n}\right)$ and $\bar{T}_{k, n}=\operatorname{ker}\left(\bar{\phi}_{n}\right)$. Since $\sum_{n=1}^{\infty} \lambda\left(\bar{I}^{n} / \bar{I}^{n-1} \bar{J}\right)-$ $e_{1}(\bar{I})=1$, there is an unique integer $N^{\prime}$ such that $\bar{T}_{N^{\prime}, n} \neq 0$ for some $n$. However, by the following remark, $\left\{\bar{a}_{0 j k} \mid j+k=t\right\}$ are not all in $\bar{I}^{N-1} \bar{J}$. Since $\sum \bar{a}_{0 j k} \bar{y}^{j} \bar{z}^{k} \in$ $\bar{I}^{N-1} \bar{J}^{t+1}$, we see that $N^{\prime}=N$.

Remark 3.3. Let $b \in I^{N} \backslash I^{N-1} J$; then $\bar{b} \notin \bar{I}^{N-1} \bar{J}$ by the fact that $I^{N}: x=I^{N-1}$.

In the sequel, let $R^{0}$ denote the set $\{$ units of $R\} \cup\{0\}$ and $R_{n}(n \geq 0)$ denote the set $\left\{f \mid f=\sum_{i=0}^{n} \lambda_{i} y^{n-i} z^{i}\right.$ for some $\left.\lambda_{i} \in R^{0}\right\}$.

Lemma 3.4. Let $(R, \mathfrak{m})$ be a 2-dimensional Cohen-Macaulay local ring with infinite residue field. Let $I$ be an $\mathfrak{m}$-primary ideal of $R$ and $J=(y, z)$ be a minimal reduction of $I$. Let $N \geq 2$. Suppose that

(i) $\lambda\left(\frac{I^{N} \cap(y)+I^{N-1} J}{I^{N-1} J}\right)=\lambda\left(\frac{I^{N} \cap(z)+I^{N-1} J}{I^{N-1} J}\right)=1$, and

(ii) $\forall n<N$ and $\forall m \geq 1, I^{n}: y=I^{n}: z=I^{n-1}$ and $I^{n} J^{m}: y=I^{n} J^{m}: z=$ $I^{n} J^{m-1}$.

Consider the exact sequence

$$
0 \longrightarrow T_{N, n} \longrightarrow \bigoplus^{n+1} I^{N} / I^{N-1} J \stackrel{\phi_{n}}{\longrightarrow} I^{N} J^{n} / I^{N-1} J^{n+1} \longrightarrow 0
$$

where $\phi_{n}=\left(y^{n}, y^{n-1} z, \ldots, z^{n}\right)$ and $T_{N, n}=\operatorname{ker}\left(\phi_{n}\right)$.

Suppose that $T_{N, n} \neq 0$ for some $n$. Let $l$ be the least integer such that $T_{N, n} \neq 0$ for all $n \geq l$. Let $a_{0}, \ldots, a_{l} \in I^{N}$ not all in $I^{N-1} J$ such that $\sum_{i=0}^{l} a_{i} y^{l-i} z^{i} \in I^{N-1} J^{l+1}$.

Then the following hold:

(1) $a_{0} \notin I^{N-1} J$ and $\mathfrak{m} a_{i} \subseteq I^{N-1} J \forall i$.

(2) If $\sum_{i=0}^{n} b_{i} y^{n-i} z^{i} \in I^{N-1} J^{n+1}$ for some $b_{i} \in I^{N}$ and for some $n$, then $\mathfrak{m} b_{i} \subseteq$ $I^{N-1} J \forall i$ and there are $\lambda_{0}, \ldots, \lambda_{n-l} \in R^{0}$ such that $b_{j}-\sum_{i=0}^{j} a_{i} \lambda_{j-i} \in I^{N-1} J$. (Conventions: $a_{i}=0$ if $i>l$ and $\lambda_{i}=0$ if $i>n-l$.)

Proof. By the choice of $l$ and the fact that $I^{N-1} J^{m}: z=I^{N-1} J^{m-1}$, we obtain that $a_{0} \notin I^{N-1} J$. Since $a_{0} \in I^{N} \cap(z)+I^{N-1} J$, we have, by assumption, $\mathfrak{m} a_{0} \subseteq I^{N-1} J$. 
Furthermore, let $w \in \mathfrak{m}$; then $\sum_{i=1}^{l}\left(w a_{i}\right) y^{l-i} z^{i-1} \in I^{N-1} J^{l}$ by the assumption that $I^{N-1} J^{m}: z=I^{N-1} J^{m-1}$. Again, by the choice of $l, w a_{i}$ must belongs to $I^{N-1} J$ for all $i$. This proves (1).

To see (2), we may assume that $n \geq l$ and $b_{0} \in I^{N} \backslash I^{N-1} J$. Then $b_{0} \in$ $I^{N} \cap(z)+I^{N-1} J$. Since $a_{0} \in I^{N} \cap(z)+I^{N-1} J$; there is a unit $\lambda_{0}$ such that $b_{0}-\lambda_{0} a_{0} \in I^{N-1} J$, therefore, $\sum_{i=0}^{n-1}\left(b_{i+1}-\lambda_{0} a_{i+1}\right) y^{n-1-i} z^{i} \in I^{N-1} J^{n}$.

If $n=l$, then, by the choice of $l, b_{i}-\lambda_{0} a_{i} \in I^{N-1} J \forall i$; hence $\mathfrak{m} b_{i} \subseteq I^{N-1} J \forall i$. If $n>l$, then by induction $\mathfrak{m} b_{i} \subseteq I^{N-1} J \forall i$ and there are $\lambda_{1}, \ldots, \lambda_{n-l} \in R^{0}$ such that $\forall j \geq 1 b_{j}-\lambda_{0} a_{j}=\sum_{i=0}^{j-1} a_{i} \lambda_{j-i}$. This proves (2).

Let $\{x, y, z\}$ be a generating set of $J$ satisfying the conditions of Proposition 3.2 Let the overbars denote $\bmod (x)$ in the following. By Lemma 2.4 it is easy to check that $\bar{R}, \bar{y}$ and $\bar{z}$ satisfy all the assumptions of Lemma 3.4. Let $l=\min \left\{n \mid \bar{T}_{N, n} \neq\right.$ $0\}$, where $\bar{T}_{N, n}$ is defined as the above; then there are $a_{0}, \ldots, a_{l} \in I^{N}$ not all in $I^{N-1} J$ such that $\sum \bar{a}_{i} \bar{y}^{l-i} \bar{z}^{i} \in \bar{I}^{N-1} \bar{J}^{l+1}$. Let $u=\sum_{i=0}^{l} a_{i} y^{l-i} z^{i}$; then $u$ has the following property.

Lemma 3.5. If $\sum_{i=0}^{n} b_{i} y^{n-i} z^{i} \in(x)+I^{N-1} J^{n+1}$ for some $b_{i} \in I^{N}$, then

(1) $\mathfrak{m} b_{i} \subseteq I^{N-1} J \forall i$.

(2) There is an $f \in R_{n-l}$ such that $\sum_{i=0}^{n} b_{i} y^{n-i} z^{i}-f u \in I^{N-1} J^{n+1}$.

Proof. By Lemma $3.4 \mathfrak{m} \bar{a}_{i} \in \bar{I}^{N-1} \bar{J}$; hence $\mathfrak{m} a_{i} \subseteq I^{N-1} J+(x) \cap I^{N} \subseteq I^{N-1} J$. Moreover, there are $\lambda_{0}, \ldots, \lambda_{n-l} \in R^{0}$ such that $\bar{b}_{j}-\sum_{i=0}^{j} \bar{a}_{i} \lambda_{j-i} \in \bar{I}^{N-1} \bar{J}$; then $b_{j}-\sum_{i=0}^{j} a_{j} \lambda_{j-i} \in I^{N-1} J+(x) \cap I^{N} \subseteq I^{N-1} J$. Therefore $\mathfrak{m} b_{i} \subseteq I^{N-1} J \forall i$. Let $f=\sum_{i=0}^{n-l} \lambda_{i} y^{n-l-i} z^{i} \in R_{n-l}$. Then

$$
\sum_{i=0}^{n} b_{i} y^{n-i} z^{i}-f u=\sum\left(b_{j}-\sum_{i=0}^{j} a_{j} \lambda_{j-i}\right) y^{n-j} z^{j} \in I^{N-1} J^{n+1} .
$$

Remark 3.6. If, in Lemma 3.5, at least one of the $b_{i}$ is not in $I^{N-1} J$, then $n \geq l$ and we can choose $f$ to be a nonzero element of $R_{n-l}$.

Since depth $G(I)=0, I^{n}: J \neq I^{n-1}$ for some $n$. Let $N^{\prime}=\min \left\{n \mid I^{n}: J \neq\right.$ $\left.I^{n-1}\right\}$. Since $I^{n}: x=I^{n-1} \forall n \leq N, N^{\prime}>N$. Let $s \notin I^{N^{\prime}-1}$ such that $s J \subseteq I^{N^{\prime}}$. 
Lemma 3.7. There exists an element $s^{\prime}$ with $s-s^{\prime} \in I^{N^{\prime}-1}$ such that $s^{\prime} y \in$ $I^{N} J^{N^{\prime}-N}, s^{\prime} z \in I^{N} J^{N^{\prime}-N}$ and $s^{\prime} x \in I^{N}(y, z)^{N^{\prime}-N}$.

Proof. Suppose we have shown for some $k>N$ that there is an element $s^{\prime}$ with $s-s^{\prime} \in I^{N^{\prime}-1}$ such that $s^{\prime} y \in I^{k} J^{N^{\prime}-k}, s^{\prime} z \in I^{k} J^{N^{\prime}-k}$ and $s^{\prime} x \in I^{k}(y, z)^{N^{\prime}-k}$. Then there are $a_{i}, b_{i j k} \in I^{k}$ such that $s^{\prime} x=\sum a_{i} y^{t-i} z^{i}$ and $s^{\prime} y=\sum b_{i j k} x^{i} y^{j} z^{k}$, where $t=N^{\prime}-k$. Therefore,

$$
\sum a_{i} y^{t-i+1} z^{i}-\sum b_{i j k} x^{i+1} y^{j} z^{k}=0 \in I^{k-1} J^{t+2} .
$$

Since $k \neq N, T_{k, t+1}=0$; therefore, $a_{i}, b_{i j k}$ are in $I^{k-1} J$. It follows that $s^{\prime} x \in$ $I^{k-1} J^{t+1}$ and $s^{\prime} y \in I^{k-1} J^{t+1}$. Similarily, we can get $s^{\prime} z \in I^{k-1} J^{t+1}$.

Finally, from the expression $s^{\prime} x \in I^{k-1} J^{t+1}$, we see that there is a $w \in I^{k-1} J^{t} \subseteq$ $I^{N^{\prime}-1}$ such that $\left(s^{\prime}-w\right) x \in I^{k-1}(y, z)^{t+1}$ and $\left(s^{\prime}-w\right) J \subseteq I^{k-1} J^{t+1}$. This completes the proof.

By Lemma 3.7 we may assume that $s$ satisfies the conditions $s J \in I^{N} J^{t}$ and $s x \in I^{N}(y, z)^{t}$, where $t=N^{\prime}-N$. Since $s \notin I^{N^{\prime}-1}, s x \notin I^{N-1} J^{t+1}$ by Lemma 2.4 Therefore, by Remark 3.6 and the condition $s x \in I^{N}(y, z)^{t}$, we obtain that $t \geq l$. Hence by Lemma 3.5, there is a nonzero element $f_{0} \in R_{t-l}$ such that

$$
s x-f_{0} u \in I^{N-1} J^{t+1} \text {. }
$$

In what follows, let $\mathbf{k}$ be the residue field of $R$. If $f=\sum_{i=0}^{n} \lambda_{i} y^{n-i} z^{i} \in R_{n}$, then we associate to $f$ a homogeneous polynomial $T(f)=F=\sum_{i=0}^{n} \bar{\lambda}_{i} Y^{n-i} Z^{i}$ in $\mathbf{k}[Y, Z]$. (Here, the overbars denote $\bmod (\mathfrak{m})$.)

Remark 3.8. From (3) and Lemma 3.5, we obtain $\mathfrak{m} s x \subseteq I^{N-1} J^{t+1}$, and therefore $\mathfrak{m} s \subseteq I^{N-1} J^{t}$. Moreover, if $f$ and $g$ are two elements of $R_{n}$ such that $F=G$, then $f-g \in \mathfrak{m} R_{n} ;$ it follows that $s f-s g \in I^{N-1} J^{n+t}$.

From (3), Lemma 3.5 and Remark 3.6 we have the following corollary.

Corollary 3.9. Let $B_{0}=\sum b_{i} y^{n-i} z^{i}$ with $b_{i} \in I^{N}$ not all in $I^{N-1} J$ and $B_{j} \in$ $(y, z)^{n-j} I^{N}$. Suppose that $B_{o}+B_{1} x+\cdots \in I^{N-1} J^{n+1}$. Then there is a nonzero element $h \in R_{n-l}$ such that $B_{0}+h u \in I^{N-1} J^{n+1}$ and $s h-f_{0}\left(B_{1}+B_{2} x+\cdots\right) \in$ $I^{N-1} J^{n+t-l}$.

Proof. By Lemma 3.5 and Remark 3.6, there is a nonzero element $h \in R_{n-l}$ such that $B_{0}+h u \in I^{N-1} J^{n+1}$. By (3), shx $-f_{0} x\left(B_{1}+B_{2} x+\cdots\right) \in I^{N-1} J^{n+t-l+1}$. It follows by Lemma 2.4 that $s h-f_{0}\left(B_{1}+B_{2} x+\cdots\right) \in I^{N-1} J^{n+t-l}$.

Let $Q_{j}(j \geq 0)$ be the set of all integers $n$ such that there exists a nonzero element $f \in R_{n}$ with $s f \in \sum_{i=0}^{j} x^{i}(y, z)^{t+n-1-i} I^{N}+I^{N-1} J^{t+n}$. (For example, since $\left.s y \in I^{N} J^{t}, 1 \in Q_{t}.\right)$

Let $m=\min \left\{j \mid Q_{j} \neq \emptyset\right\}$. Let $k$ be the smallest integer in $Q_{m}$; then there is a nonzero element $f \in R_{k}$ with $s f \in \sum_{i=0}^{m} x^{i}(y, z)^{t+k-1-i} I^{N}+I^{N-1} J^{t+k}$, so that 
there are $A_{i} \in(y, z)^{t+k-1-i} I^{N}$ such that

$$
s f-\sum_{i=0}^{m} A_{i} x^{i} \in I^{N-1} J^{t+k} .
$$

Lemma 3.10. Let $C_{i} \in(y, z)^{n-i} I^{N}$ such that $\sum_{i=0}^{m} C_{i} x^{i} \in I^{N-1} J^{n+1}$. Then $C_{i} \in$ $I^{N-1} J^{n+1-i}$ and all the coefficients of $C_{i}$ are all in $I^{N-1} J$.

Proof. If $m=0$, then $C_{0} \in I^{N-1} J^{n+1}$. If the coefficients of $C_{0}$ are not all in $I^{N-1} J$, then by Corollary 3.9 there is a nonzero element $h \in R_{n-l}$ such that $s h \in I^{N-1} J^{n+t-l}$. This gives the contradiction $s \in I^{N-1} J^{t}$ by Lemma 2.4.

Assume that $m \geq 1$ and the assertion is false. Let $j$ be the least integer such that the coefficients of $C_{j}$ are not all in $I^{N-1} J$. Then, by Lemma $2.4 \sum_{i=j}^{m} C_{i} x^{i-j} \in$ $I^{N-1} J^{n+1-j}$; hence by Corollary 3.9 there is a nonzero element $h \in R_{n-j-l}$ such that $s h-f_{0}\left(\sum_{i=j+1}^{m} C_{i} x^{i-j-1}\right) \in I^{N-1} J^{n+t-j-l}$, which contradicts the choice of $m$. The assertion now follows.

Let $p$ be the maximal integer such that $Z^{p} \mid F$. Let $F^{\prime}=F / Z^{p}$.

Lemma 3.11. If $g$ is a nonzero element of $R_{n}$ such that $s g \in \sum_{i=0}^{m} x^{i}(y, z)^{t+n-1-i} I^{N}$ $+I^{N-1} J^{t+n}$, then $F \mid G$.

Proof. Let $B_{i} \in(y, z)^{t+n-1-i} I^{N}$ such that

$$
s g-\sum_{i=0}^{m} B_{i} x^{i} \in I^{N-1} J^{t+n} .
$$

Write $G=G^{\prime} Z^{q}$ with $\left(G^{\prime}, Z\right)=1$. Let $g^{\prime} \in R_{n-q}$ and $f^{\prime} \in R_{k-p}$ such that $T\left(g^{\prime}\right)=G^{\prime}$ and $T\left(f^{\prime}\right)=F^{\prime}$. By Remark 3.8, we may assume that $f=f^{\prime} z^{p}$ and $g=g^{\prime} z^{q}$.

Assume that $q<p$. Then from (44) and (5), we obtain that

$$
\sum_{i=0}^{m}\left(f^{\prime} z^{p-q} B_{i}-g^{\prime} A_{i}\right) x^{i} \in I^{N-1} J^{t+k+n-q}
$$

so that by Lemma 3.10 all the coefficients of $f^{\prime} z^{p-q} B_{i}-g^{\prime} A_{i}$ are all in $I^{N-1} J$. Since $\left(G^{\prime}, Z\right)=1$, there are $A_{i}^{\prime} \in(y, z)^{t+k-2-i} I^{N}$ such that $A_{i}-z A_{i}^{\prime} \in I^{N-1} J^{t+k-i}$; it follows from (44) that $s\left(f^{\prime} z^{p-1}\right)-\sum_{i=0}^{m} A_{i}^{\prime} x^{i} \in I^{N-1} J^{t+k-1}$, which contradicts the choice of $k$. Hence $q \geq p$.

Write $G=G^{\prime} Z^{p}$. Then $G^{\prime}=F^{\prime} Q+Z^{n-k+1} G^{\prime \prime}$ for some $Q$ and $G^{\prime \prime}$. Suppose $G^{\prime \prime} \neq 0$. Then $\operatorname{deg} G^{\prime \prime}=\operatorname{deg} F^{\prime}-1$. Let $g^{\prime \prime} \in R_{k-p-1}$ such that $T\left(g^{\prime \prime}\right)=G^{\prime \prime}$; then

$$
s g^{\prime \prime} z^{n-k+1+p}-\sum_{i=0}^{m} C_{i} x^{i} \in I^{N-1} J^{t+n}
$$

for some $C_{i} \in(y, z)^{t+n-1-i} I^{N}$. 
From (4) and (7), we obtain that

$$
\sum_{i=0}^{m}\left(g^{\prime \prime} z^{n-k+1} A_{i}-f^{\prime} C_{i}\right) x^{i} \in I^{N-1} J^{t+n+k-p}
$$

therefore, by Lemma 3.10 all the coefficients of $g^{\prime \prime} z^{n-k+1} A_{i}-f^{\prime} C_{i}$ are in $I^{N-1} J$. Since $\left(F^{\prime}, Z\right)=1$, there are $C_{i}^{\prime} \in(y, z)^{t+k-2-i} I^{N}$ such that $C_{i}-z^{n-k+1} C_{i}^{\prime} \in$ $I^{N-1} J^{t+n-i}$. Hence from (7) $s g^{\prime \prime} z^{p}-\sum_{i=0}^{m} C_{i}^{\prime} x^{i} \in I^{N-1} J^{t+k-1}$, which contradicts the choice of $k$. Therefore, $G^{\prime \prime}=0$ and $F \mid G$.

In fact, Lemma 3.11 can be improved as follows.

Lemma 3.12. Let $m \leq m^{\prime} \leq t$. If $g$ is a nonzero element of $R_{n}$ such that $s g \in$ $\sum_{i=0}^{m^{\prime}} x^{i}(y, z)^{t+n-1-i} I^{N}+I^{N-1} J^{t+n}$, then $F \mid G$.

Proof. We use induction on $m^{\prime}$. If $m^{\prime}=m$, then this is the content of Lemma 3.11 Assume that $m^{\prime}>m$. Let $H_{0}=\frac{F_{0}}{\left(F_{0}, F\right)}$ and let $n_{0}=\operatorname{deg} H_{0}$. Let $h_{0} \in R_{n_{0}}$ such that $T\left(h_{0}\right)=H_{0}$. Let $B_{i} \in(y, z)^{t+n-1-i} I^{N}$ such that

$$
s g-\sum_{i=0}^{m^{\prime}} B_{i} x^{i} \in I^{N-1} J^{t+n}
$$

Let $H=(F, G)$ and let $k^{\prime}=\operatorname{deg} H$. Then $F=F^{\prime} H$ and $G=G^{\prime} H$ for some $F^{\prime}$ and $G^{\prime}$. Let $f^{\prime} \in R_{k-k^{\prime}}$ and $g^{\prime} \in R_{n-k^{\prime}}$ such that $T\left(f^{\prime}\right)=F^{\prime}$ and $T\left(g^{\prime}\right)=G^{\prime}$. By Remark 3.8, we may assume that $f=f^{\prime} h$ and $g=g^{\prime} h$. Set $A_{i}=0 \forall i>m$. From (4) and (8), we obtain that $\sum_{i=0}^{m^{\prime}}\left(f^{\prime} B_{i}-g^{\prime} A_{i}\right) x^{i} \in I^{N-1} J^{t+n+k-k^{\prime}}$. Therefore, by Lemma 3.5 there is an element $h_{1} \in R_{t+n+k-k^{\prime}-l-1}$ such that $f^{\prime} B_{0}-g^{\prime} A_{0}+$ $h_{1} u \in I^{N-1} J^{t+n+k-k^{\prime}}$, and then, by (3), sh $h_{1}-f_{0}\left(\sum_{i=0}^{m^{\prime}-1}\left(f^{\prime} B_{i+1}-g^{\prime} A_{i+1}\right) x^{i}\right) \in$ $I^{N-1} J^{2 t+n+k-k^{\prime}-l-1}$. Hence, by induction, $F \mid H_{1}$. Moreover, since $\left(F_{0}, F\right)$ is a common factor of $H_{1}$ and $F_{0}$, there is an element $g_{1} \in R_{n_{0}+n+k-k^{\prime}-1}$ such that $s g_{1}-h_{0}\left(\sum_{i=0}^{m^{\prime}-1}\left(f^{\prime} B_{i+1}-g^{\prime} A_{i+1}\right) x^{i}\right) \in I^{N-1} J^{t+n_{0}+n+k-k^{\prime}-1}$. Therefore, by induction, $F \mid G_{1}$. Hence there is an element $g_{1}^{\prime} \in R_{n_{0}+n-k^{\prime}-1}$ such that $G_{1}=G_{1}^{\prime} F$.

Suppose that $m=0$. Since $f^{\prime} B_{0}-g^{\prime} A_{0}+h_{1} u \in I^{N-1} J^{t+n+k-k^{\prime}}$, by Lemma 3.10 . $g^{\prime} h A_{o}-f A_{0}^{\prime} \in I^{N-1} J^{t+n+k}$ for some $A_{0}^{\prime} \in(y, z)^{t+n-1} I^{N}$. Therefore by (4), sf $g^{\prime} h-$ $f A_{0}^{\prime} \in I^{N-1} J^{t+n+k}$, and then $s g^{\prime} h-A_{0}^{\prime} \in I^{N-1} J^{t+n}$ by Lemma 2.4 so that by Lemma 3.11, $F \mid G^{\prime} H$. But $\left(F, G^{\prime}\right)=1$; we obtain $F \mid H$.

Assume now that $m \geq 1$. We claim: There are integers $n_{1}, \ldots, n_{m^{\prime}-m}, d_{1}, \ldots$, $d_{m^{\prime}-m}$ and elements $h_{j} \in R_{n_{j}}, g_{j} \in R_{d_{j}}$ and $g_{j}^{\prime} \in R_{d_{j}-k}$ satisfy the following conditions:

(i) $G_{j}=G_{j}^{\prime} F$.

(ii) $h_{0}^{j-1}\left(f^{\prime} B_{j-1}-g^{\prime} A_{j-1}\right)+h_{0}^{j-2} g_{1}^{\prime} A_{j-2}-\cdots-g_{j-1}^{\prime} A_{0}+h_{j} u \in I^{N-1} J^{n_{j}+l+1}$. 
(iii) $s g_{j}-\sum_{i=0}^{m^{\prime}-j}\left(h_{0}^{j}\left(f^{\prime} B_{i+j}-g^{\prime} A_{i+j}\right)-h_{0}^{j-1} g_{1}^{\prime} A_{i+j-1}-\cdots-h_{0} g_{j-1}^{\prime} A_{i+1}\right) x^{i} \in$ $I^{N-1} J^{d_{j}+t}$.

Suppose that we have constructed, for some $j \geq 1, h_{j}, g_{j}$ and $g_{j}^{\prime}$. Then from (i), (iii) and (4), we see that the element

$$
\begin{aligned}
& h_{0}^{j}\left(f^{\prime} B_{j}-g^{\prime} A_{j}\right)-h_{0}^{j-1} g_{1}^{\prime} A_{j-1}-\cdots-g_{j}^{\prime} A_{0} \\
& \quad+\sum_{i=0}^{m^{\prime}-j-1}\left(h_{0}^{j}\left(f^{\prime} B_{i+j+1}-g^{\prime} A_{i+j+1}\right)-h_{0}^{j-1} g_{1}^{\prime} A_{i+j}-\cdots-g_{j}^{\prime} A_{i+1}\right) x^{i+1}
\end{aligned}
$$

is in $I^{N-1} J^{d_{j}+t}$. Therefore, by Lemma 3.5, there is an element $h_{j+1} \in R_{n_{j+1}}$ for some $n_{j+1}$ such that (ii) holds for $j+1$ and $F \mid H_{j+1}$ (cf. the construction of $h_{1}$ ) by induction. Moreover, from the construction of $g_{1}$, it is easy to see that there is an element $g_{j+1} \in R_{d_{j+1}}$ for some $d_{j+1}$ such that (iii) holds for $j+1$. Since by induction $F \mid G_{j+1}$, there is an $g_{j+1}^{\prime} \in R_{d_{j+1}-k}$ such that (i) holds. This proves the claim.

Set $j=m^{\prime}-m$ in (iii) of the claim and compare with (4). We obtain that the element

$\sum_{i=0}^{m}\left(h_{0}^{m^{\prime}-m}\left(f^{\prime} B_{i+m^{\prime}-m}-g^{\prime} A_{i+m^{\prime}-m}\right)-h_{0}^{m^{\prime}-m-1} g_{1}^{\prime} A_{i+m^{\prime}-m-1}-\cdots-g_{m^{\prime}-m}^{\prime} A_{i}\right) x^{i}$

is in $I^{N-1} J^{d_{m^{\prime}-m}+t}$. However by Lemma 3.10 $\forall i \leq m$,

$$
\begin{array}{r}
h_{0}^{m^{\prime}-m}\left(f^{\prime} B_{i+m^{\prime}-m}-g^{\prime} A_{i+m^{\prime}-m}\right)-h_{0}^{m^{\prime}-m-1} g_{1}^{\prime} A_{i+m^{\prime}-m-1}-\cdots-g_{m^{\prime}-m}^{\prime} A_{i} \\
\in I^{N-1} J^{d_{m^{\prime}-m}+t-i}
\end{array}
$$

From (ii) and (9), it is not hard to see that for $0 \leq i \leq m$ there are $A_{i}^{\prime} \in$ $(y, z)^{e_{i}} I^{N}$ for some $e_{i}$ such that $h h_{0}^{i} g^{i+1} A_{i}-f A_{i}^{\prime} \in I^{N-1} J^{e_{i}+k+1}$. Therefore by (4), $s h h_{0}^{m} g^{\prime m+1} f-f\left(\sum_{i=0}^{m} h_{0}^{m-i} g^{\prime m-i} A_{i}^{\prime} x^{i}\right) \in I^{N-1} J^{e}$ for some $e$; it follows by Lemma 2.4 that

$$
s h h_{0}^{m} g^{\prime m+1}-\sum_{i=0}^{m} h_{0}^{m-i} g^{\prime m-i} A_{i}^{\prime} x^{i} \in I^{N-1} J^{e-k} .
$$

Finally, by Lemma 3.11, $F \mid G^{\prime m+1} H H_{0}^{m}$. Since $\left(F, G^{\prime}\right)=\left(F, H_{0}\right)=1$, we have $F \mid H$. This completes the proof.

Now, choose a unit $\lambda$ so that $(Y+\lambda Z, F)=1$. Since $s(y+\lambda z) \in \sum_{i=0}^{t} x^{i}(y, z)^{t-i} I^{N}+$ $I^{N-1} J^{t+1}$, by Lemma 3.12, $F \mid Y+\lambda Z$, which contradicts the choice of $\lambda$. This proves Theorem 3.1 .

By Proposition 2.6] and Theorem[3.1, we have the following corollary.

Corollary 3.13. Let $(R, \mathfrak{m})$ be a Cohen-Macaulay local ring of dimension $d \geq 2$ with infinite residue field. Let $I$ be an $\mathfrak{m}$-primary ideal of $R$. Suppose that there is a minimal reduction $J$ of $I$ such that $\sum_{n=1}^{\infty} \lambda\left(I^{n} \cap J / I^{n-1} J\right)=2$. Then depth $G(I) \geq$ $d-2$. 


\section{ACKNOWLEDGMENT}

I would like to thank the referee for a very careful reading of the paper, and many valuable comments, which improved the exposition a lot.

\section{REFERENCES}

1. A. Guerrieri, On the depth of the associated graded ring of an m-primary ideal of a CohenMacaulay local ring, J. Algebra. 167 (1994), 745-757. MR 95h:13004

2. MR 95c: 13002

3. S. Huckaba, A d-dimensional extension of a lemma of Huneke's and formulas for the Hilbert coefficients, Proc. Amer. Math. Soc. 124 (1996), 1393-1401. MR 96g:13018

4. S. Huckaba and T. Marley, Hilbert coefficients and the depth of associated graded rings, J. London Math. soc.(2) 56 (1997), no. 1, 64-76. MR 98i:13028

5. J. D. Sally, Super-regular sequences, Pacific J. Math. 84 (1979), 465-481. MR 81m:13024

6. P. Valabrega and G.Valla, Form rings and regular sequence, Nagoya Math. J. 72 (1978), 93101. MR 80d:14010

7. W. V. Vasconcelos, Hilbert functions, analytic spread, and Koszul homology, Contemporary Math. 159 (1994), 401-422. MR 95a:13006

8. H.-J Wang, An interpretation of depth $G(I)$ and $e_{1}(I)$ via the Sally module, Communications in Algebra 25,(1) (1997), 303-309. MR 98a:13035

Department of Mathematics, National Chung Cheng University, Minghsiung, Chiayi 621, TAIWAN

E-mail address: hjwang@math.ccu.edu.tw 